\title{
View-Independent Enhanced 3D Reconstruction of Non-Rigidly Deforming Objects
}

\author{
Hassan Afzal ${ }^{1}$, Djamila Aouada ${ }^{1}$, François Destelle ${ }^{2}$, Bruno Mirbach $^{3}$, and Björn \\ Ottersten $^{1}$ \\ 1 Interdisciplinary Centre for Security, Reliability and Trust \\ University of Luxembourg, 4, rue Alphonse Weicker, L-2721, Luxembourg \\ Email: \{hassan.afzal, djamila.aouada, bjorn.ottersten\}@uni.lu \\ 2 Dublin City University, Insight: Centre for Data Analytics, Ireland \\ Email: francois.destelle@dcu.ie \\ 3 IEE S.A., Advanced Engineering, Contern, Luxembourg \\ Email: bruno.mirbach@iee.lu
}

\begin{abstract}
In this paper, we target enhanced 3D reconstruction of non-rigidly deforming objects based on a view-independent surface representation with an automated recursive filtering scheme. This work improves upon the KinectDeform algorithm which we recently proposed. KinectDeform uses an implicit viewdependent volumetric truncated signed distance function (TSDF) based surface representation. The view-dependence makes its pipeline complex by requiring surface prediction and extraction steps based on camera's field of view. This paper proposes to use an explicit projection-based Moving Least Squares (MLS) surface representation from point-sets. Moreover, the empirical weighted filtering scheme in KinectDeform is replaced by an automated fusion scheme based on a Kalman filter. We analyze the performance of the proposed algorithm both qualitatively and quantitatively and show that it is able to produce enhanced and feature preserving $3 \mathrm{D}$ reconstructions.
\end{abstract}

\section{Introduction}

Data acquired by commodity 3D sensing technologies is noisy and of limited resolution. This limits its direct use in applications ranging from environment mapping for mobile autonomous systems and preservation of historical sites, to human activity and gesture recognition for virtual communications, assistive robotics, security and surveillance.

Research has been carried out to build techniques around commodity 3D sensing technologies to accurately reconstruct captured 3D objects or scenes by relying on training data or use of templates such as in the case of [13] and [25] or by fusing a specified number of captured frames to produce a single high quality 3D reconstruction [19]. KinectFusion and similar techniques provide an effective and efficient mechanism to recursively fuse and filter the incoming information to produce enhanced 3D reconstructions of the environment [15], [17]. The downside of these techniques is that they lack the ability to tackle the non-rigid behavior of deforming objects [4], [16], [20]. Some of these techniques. e.g., for human face modeling and full-body 3D reconstruction, are restricted to very limited non-rigid behavior and require subjects to remain as 
rigid as possible [14], [6], [21]. To tackle these issues researchers have proposed other methods such as [27], [26] and [12], which use high quality pre-built templates or construct them as a first step and use them to track the non-rigidities and provide accurate and complete $3 \mathrm{D}$ recontructions.

Recently, researchers have focused on tracking highly non-rigid behaviors of deforming objects without the knowledge of any prior shape or reference [18], [7], for the purposes of, for example, depth video enhancement [9]. In our previous work, known as KinectDeform, we showed that a non-rigid registration method can be used in a recursive pipeline similar to KinectFusion to produce enhanced 3D reconstructions of deforming objects [2]. The non-rigid registration step in the pipeline is followed by surface filtering or fusion using volumetric truncated signed distance function (TSDF) based implicit surface representation. This surface representation scheme is view-dependent and requires organized point clouds as input. Since non-rigid registration deforms and hence destroys the organization of input point clouds, an expensive data-reorganization step in the form of meshing and ray-casting is required before surface fusion. Moreover, for fusion, a weighted average scheme is used for which parameters are chosen empirically for each iteration. Ray-casting is used again to extract the resulting point-based surface from fused $T S D F$ volumes after every iteration.

In this paper, we propose a method called View-Independent KinectDeform (VI-KinectDeform) which improves upon the KinectDeform algorithm by replacing the volumetric TSDF based view-dependent surface representation with an octree-based viewindependent and explicit surface representation using Point Set Surfaces based on the method of Moving Least Squares [3]. This results in a simplified version of KinectDeform with the removal of an expensive data reorganization step. Moreover, we also improve upon the fusion mechanism by proposing an automated recursive filtering scheme using a simple Kalman filter [10]. Due to our explicit surface representation, surface prediction step at the end of each iteration is also not required resulting in a simpler algorithm. We compare the results of VI-KinectDeform with those of KinectDeform using non-rigidly deforming objects and show that for the same number of iterations VI-KinectDeform produces stable and more accurate 3D reconstructions.

The remainder of this paper is organized as follows: Section 2 describes the problem at hand and gives a background on the surface representation and recursive filtering method proposed in KinectDeform. This is followed by an introduction to the Point Set Surfaces based on MLS. Section 3 details the proposed approach. Section 4 presents qualitative and quantitative evaluation of results of the proposed method and compares them with the results of KinectDeform and other methods. This is followed by a conclusion in Sect. 5.

\section{Background}

\subsection{Problem Formulation and KinectDeform}

At each discrete time-step $i \in \mathbb{N}$, a static or moving camera acquires a point cloud $\mathcal{V}_{i}$ containing a number of points $U \in \mathbb{N}$. Note that $\mathcal{V}_{i}$ may be organized or unorganized. The point-set $\left\{\mathbf{p}_{j}\right\}$ in $\mathcal{V}_{i}$, where $\mathbf{p}_{j} \in \mathbb{R}^{3}$ and $j \in\{1, \ldots, U\}$, approximates the underlying surface of deformable objects in camera's field of view. Considering a sequence 
of $N$ such acquired point clouds $\left\{\mathcal{V}_{0}, \mathcal{V}_{1}, \ldots, \mathcal{V}_{N-1}\right\}$, each acquisition $\mathcal{V}_{i}$ is associated with the previous acquisition $\mathcal{V}_{i-1}$ via [2]:

$$
\mathcal{V}_{i}=h_{i}\left(\mathcal{V}_{i-1}\right)+\mathcal{E}_{i},
$$

where $h_{i}(\cdot)$ is the non-rigid deformation which deforms $\mathcal{V}_{i-1}$ to $\mathcal{V}_{i}$, and $\mathcal{E}_{i}$ represents the sensor noise and sampling errors. The problem at hand is therefore to reduce $\mathcal{E}_{i}$ for $i>0$, to recover an enhanced sequence $\left\{\mathcal{V}_{0}^{f^{\prime}}, \mathcal{V}_{1}^{f^{\prime}}, \ldots, \mathcal{V}_{N-1}^{f^{\prime}}\right\}$ starting from the input sequence $\left\{\mathcal{V}_{0}, \mathcal{V}_{1}, \ldots, \mathcal{V}_{N-1}\right\}$ [2]. In KinectDeform, we defined a recursive filtering function $f(\cdot, \cdot)$ to solve this problem which sequentially fuses the current measurement $\mathcal{V}_{i}$ with the result of the previous iteration $\mathcal{V}_{i-1}^{f^{\prime}}$ by tracking the non-rigid deformations between them such that:

$$
\mathcal{V}_{i}^{f^{\prime}}= \begin{cases}\mathcal{V}_{i} & \text { for } i=0 \\ f\left(\mathcal{V}_{i-1}^{f^{\prime}}, \mathcal{V}_{i}\right) & i>0\end{cases}
$$

As mentioned before a major shortcoming of the KinectDeform scheme lies in the 3D surface representation based on the view-dependent truncated signed distance function $(T S D F)$ volume for data fusion and filtering [2]. Construction of a TSDF volume for a point cloud $\mathcal{V}_{i}$ requires computing a scalar TSDF value for each voxel represented by its centroid $\mathbf{c} \in \mathbb{R}^{3}$. The $T S D F$ function $S_{\mathcal{V}_{i}}$ may be defined as follows:

$$
S_{\mathcal{V}_{i}}(\mathbf{c})=\Psi\left(\|\mathbf{c}\|_{2}-\left\|\mathbf{p}_{j}\right\|_{2}\right),
$$

where $j=\pi(\mathbf{K c}), j \in\{1, \ldots, U\}$, is projection of the centroid $\mathbf{c}$ to camera's image plane using camera's intrinsic matrix $\mathbf{K}$. This, in turn, requires the points in $\mathcal{V}_{i}$ to be organized with respect to the image plane, moreover:

$$
\Psi(\eta)= \begin{cases}\min \left\{1, \frac{\eta}{\mu}\right\} \cdot \operatorname{sgn}(\eta) & \text { iff } \eta \geq-\mu \\ 0 & \text { otherwise }\end{cases}
$$

where $\mu$ is the truncation distance and $\operatorname{sgn}$ is the sign function. Therefore, after nonrigid registration which destroys the data organization of our input point $\operatorname{cloud} \mathcal{V}_{i}^{f^{\prime}}$, an expensive data reorganization step based on meshing and ray-casting is required for computation of a TSDF. After that, the TSDF volumes created using $\mathcal{V}_{i}^{r}$ and $\mathcal{V}_{i}$ are fused together using an empirical weighting scheme whereby the weighting parameters are chosen manually. This is followed by another surface prediction step via ray-casting to extract the final filtered surface from the fused volume.

\subsection{Point Set Surfaces}

Keeping in view the KinectDeform method explained in Sect. 2.1, a simpler approach would be to replace the view-dependent TSDF volume-based surface representation for fusion and filtering with a view-independent surface representation. This would result in avoiding data reorganization and surface prediction steps. As mentioned before the input points $\left\{\mathbf{p}_{j}\right\}$ approximate the underlying surface of objects in the scene. In [3], Alexa et al. built upon Levin's work [11], and proposed a view-independent point-based 
surface reconstruction method based on Moving Least Squares (MLS). This method projects a point $\mathbf{q}$ lying near $\left\{\mathbf{p}_{j}\right\}$ on the underlying surface approximated by the local neighborhood of $\mathbf{q}$. Apart from facilitating the computation of the differential geometric properties of the surface such as normals and curvatures, this method is able to handle noisy data and provides smooth reconstructions. Moreover, the local nature of projection procedure improves the efficiency of the algorithm [5].

The projection procedure as proposed by Alexa et al. is divided into two steps [3]. In the first step a local reference domain, i.e., a plane $H_{\mathbf{q}}=\left\{\mathbf{p} \in \mathbb{R}^{3}: \mathbf{n}^{T} \mathbf{p}=\mathbf{n}^{T} \mathbf{v}\right\}$, $\mathbf{v}, \mathbf{n} \in \mathbb{R}^{3}$ and $\|\mathbf{n}\|=1$, is computed by minimizing the following non-linear energy function [5]:

$$
e_{M L S}(\mathbf{v}, \mathbf{n})=\sum_{s_{\mathbf{q}}=1}^{U_{\mathbf{q}}} w\left(\left\|\mathbf{p}_{s_{\mathbf{q}}}-\mathbf{v}\right\|\right)\left\langle\mathbf{n}, \mathbf{p}_{s_{\mathbf{q}}}-\mathbf{v}\right\rangle^{2},
$$

where $\left\{\mathbf{p}_{s_{\mathbf{q}}}\right\} \subset\left\{\mathbf{p}_{j}\right\}, s_{\mathbf{q}} \in\left\{1, \ldots, U_{\mathbf{q}}\right\}$ and $U_{\mathbf{q}}$ is the total number of neighboring points within a fixed radius around $\mathbf{q}$. Also $\mathbf{n}=(\mathbf{q}-\mathbf{v}) /\|\mathbf{q}-\mathbf{v}\|,\langle.,$.$\rangle is the dot$ product and $w(e)=\exp ^{\left(-\frac{e^{2}}{d^{2}}\right)}$ is the Gaussian weight function where $d$ represents the anticipated spacing between neighboring points [3]. The surface features of size less than $d$ are smoothed out due to the $M L S$ projection. Replacing $\mathbf{v}$ by $\mathbf{q}+t \mathbf{n}$ where $t \in \mathbb{R}$ in (5) we have:

$$
e_{M L S}(\mathbf{q}, \mathbf{n})=\sum_{s_{\mathbf{q}}=1}^{U_{\mathbf{q}}} w\left(\left\|\mathbf{p}_{s_{\mathbf{q}}}-\mathbf{q}-t \mathbf{n}\right\|\right)\left\langle\mathbf{n}, \mathbf{p}_{s_{\mathbf{q}}}-\mathbf{q}-t \mathbf{n}\right\rangle^{2} .
$$

The minimum of (6) is found with the smallest $t$ and the local tangent plane $H_{\mathbf{q}}$ near $\mathbf{q}[3]$. The local reference domain is then defined by an orthonormal coordinate system in $H_{\mathbf{q}}$ with $\mathbf{v}$ as its origin [5].

In the next step, we find the orthogonal projections of points in $\left\{\mathbf{p}_{s_{\mathrm{v}}}\right\} \subset\left\{\mathbf{p}_{j}\right\}$, where $s_{\mathbf{v}} \in\left\{1, \ldots, U_{\mathbf{v}}\right\}$, lying in the local neighborhood of $\mathbf{v}$ to get their corresponding 2D representations $\left(x_{s_{\mathbf{v}}}, y_{s_{\mathbf{v}}}\right)$ in the local coordinate system in $H_{\mathbf{q}}$. The height of $\mathbf{p}_{s_{\mathbf{v}}}$ over $H_{\mathbf{q}}$ is found via:

$$
h_{s_{\mathbf{v}}}=\left\langle\mathbf{n}, \mathbf{p}_{s_{\mathbf{v}}}-\mathbf{q}-t \mathbf{n}\right\rangle .
$$

Using the local 2D projections and the height map, a local bivariate polynomial approximation $g: \mathbb{R}^{2} \rightarrow \mathbb{R}$ is computed by minimizing the weighted least squares error:

$$
\sum_{s_{\mathbf{v}}=1}^{U_{\mathbf{v}}} w\left(\left\|\mathbf{p}_{s_{\mathbf{v}}}-\mathbf{q}-t \mathbf{n}\right\|\right)\left(g\left(x_{s_{\mathbf{v}}}, y_{s_{\mathbf{v}}}\right)-h_{s_{\mathbf{v}}}\right)^{2} .
$$

The degree of the polynomial to be computed is fixed beforehand. At the end, projection $P$ of $\mathbf{q}$ onto the underlying surface is defined by the polynomial value at the origin, i.e.:

$$
P(\mathbf{q})=\mathbf{v}+g(0,0) \mathbf{n}=\mathbf{q}+(t+g(0,0)) \mathbf{n} .
$$

The projected point is considered to be the resulting filtered point lying on the approximated surface. These two steps are repeated for all points which need to be sampled to sufficiently represent the surfaces of objects in camera's field of view to get enhanced $3 \mathrm{D}$ reconstructions. 


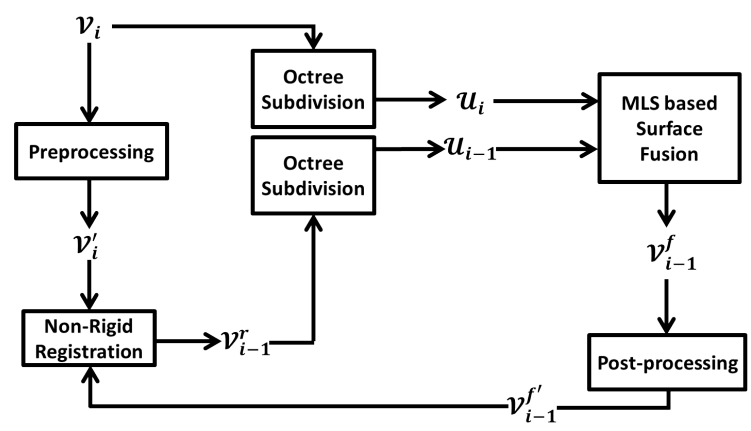

Fig. 1. Detailed pipeline of VI-KinectDeform. $\mathcal{V}_{i}$ : input point cloud at time-step $i$. $\mathcal{V}_{i}^{\prime}$ : result of pre-processing on $\mathcal{V}_{i} . \mathcal{V}_{i}^{r}$ : result of non-rigid registration of $\mathcal{V}_{i-1}^{f^{\prime}}$ to $\mathcal{V}_{i}^{\prime} . \mathcal{U}_{i}$ and $\mathcal{U}_{i-1}$ : resulting voxel sets based on octree sub-division corresponding to $\mathcal{V}_{i}$ and $\mathcal{V}_{i-1}^{r}$ respectively. $\mathcal{V}_{i-1}^{f}$ : the result of projection-based $M L S$ surface computation and Kalman filtering-based fusion. $\mathcal{V}_{i-1}^{f}$ : the final result after post-processing. For more details please read Sects. 2 and 3.

\section{Proposed Technique}

Figure 1 shows the pipeline of VI-KinectDeform which is an improved/simplified version of KinectDeform. After the non-rigid registration step which deforms $\mathcal{V}_{i-1}^{f^{\prime}}$ to produce $\mathcal{V}_{i-1}^{r}$ which is mapped to $\mathcal{V}_{i}$, the data reorganization step is removed. Instead, a view-independent surface representation and filtering based on the $M L S$ method is proposed. Since the $M L S$ method works on the local neighborhoods of sampled points, voxelizing/sub-dividing the space of input 3D point clouds not only provides us with sampling information but also helps in accelerating the search for local neighborhoods of the sampled points. After that, the sampled points are projected onto the underlying surfaces of both point clouds based on the $M L S$ method. The resulting projections are then fused together via an automatic Kalman filtering based scheme to give enhanced $3 \mathrm{D}$ reconstructions. These steps are explained as follows:

\subsection{Sampling and $M L S$ based projection}

We use octree data structure to sample the space occupied by $\mathcal{V}_{i}$ and $\mathcal{V}_{i-1}^{r}$ resulting in two voxel sets $\mathcal{U}_{i}$ and $\mathcal{U}_{i-1}$ with a pre-defined depth $l \in \mathbb{N}$. At depth level $l, \mathcal{U}_{i}$ and $\mathcal{U}_{i-1}$ contain $m_{i}^{l}$ and $m_{i-1}^{l}$ non-empty voxels, respectively. It is to be noted that since $\mathcal{V}_{i}$ and $\mathcal{V}_{i-1}^{r}$ are mapped, the corresponding voxels in $\mathcal{U}_{i}$ and $\mathcal{U}_{i-1}$ occupy the same space. Each voxel $u_{i, a}^{l} \in \mathcal{U}_{i}$ where $a \in\left\{1, \ldots, m_{i}^{l}\right\}$ (or similarly each voxel $u_{i-1, b}^{l} \in \mathcal{U}_{i-1}$ ) is represented by its geometric center $\mathbf{c}_{i, a}^{l}$ (or $\mathbf{c}_{i-1, b}^{l}$ ), the points contained in the voxel and information about its immediate neighbors. These centroids lying near input points provide us with suitable sampling points to be projected onto the underlying surface based on the procedure explained in Sect. 2.2. Therefore, in the next step the centroid of each non-empty leaf voxel in $\mathcal{U}_{i} \cup \mathcal{U}_{i-1}$ lying in the vicinity of points from both $\mathcal{V}_{i}$ 
and $\mathcal{V}_{i-1}^{r}$ is projected on the approximated underlying surfaces using its corresponding neighborhood points in $\mathcal{V}_{i}$ and $\mathcal{V}_{i-1}^{r}$ respectively via the $M L S$ method to get:

$$
\begin{gathered}
\mathbf{p}_{i, k}=P_{i}\left(\mathbf{c}_{i, a}^{l}\right), \mathbf{p}_{i-1, k}=P_{i-1}\left(\mathbf{c}_{i, a}^{l}\right), \text { or } \\
\mathbf{p}_{i, k}=P_{i}\left(\mathbf{c}_{i-1, b}^{l}\right), \mathbf{p}_{i-1, k}=P_{i-1}\left(\mathbf{c}_{i-1, b}^{l}\right) .
\end{gathered}
$$

The degree of the bivariate polynomial approximating the underlying surface computed for each centroid is kept variable (max. 3 for our experiments) depending on the number of points found in the neighborhood. Hence as a result of the $M L S$-based projection procedure, two sets of corresponding filtered points, $\left\{\mathbf{p}_{i, k}\right\}$ and $\left\{\mathbf{p}_{i-1, k}\right\}$, are generated.

\subsection{Fusion}

It is clear that under ideal conditions, i.e., sensor noise free and with perfectly registered inputs $\mathcal{V}_{i}$ and $\mathcal{V}_{i-1}^{r},\left\{\mathbf{p}_{i, k}\right\}$ and $\left\{\mathbf{p}_{i-1, k}\right\}$ should be same. Therefore in this step we propose a methodology to fuse the corresponding projected points $\left\{\mathbf{p}_{i, k}\right\}$ and $\left\{\mathbf{p}_{i-1, k}\right\}$, taking into account noise factors affecting them to produce a filtered 3D reconstruction $\mathcal{V}_{i}^{f}$. In KinectDeform we performed a surface fusion/filtering using a weighted average of TSDF values of corresponding voxels [2]. The weights are chosen empirically based on an analysis of noise factors affecting the two input voxel sets per iteration. The main noise factor affecting the current measurement $\mathcal{V}_{i}$, and hence $\left\{\mathbf{p}_{i, k}\right\}$, is the sensor noise while on the other hand for $\mathcal{V}_{i-1}^{r}$ it is assumed that, due to pre-processing, some amount of this sensor noise is mitigated with some loss of details and hence the main noise factor is error due to non-rigid registration [2]. This should be coupled with iterative effects of filtering as $\mathcal{V}_{i-1}^{r}$ is indeed a deformed state of the filtered $\mathcal{V}_{i-1}^{f^{\prime}}$.

To tackle these factors, we propose an automatic filtering approach by point tracking with a Kalman filter [10]. The observation model is based on the current measurements i.e. $\left\{\mathbf{p}_{i, k}\right\}$, and the associated sensor noise $n_{i}^{s}$ is assumed to follow a Gaussian distribution $n_{i}^{s} \sim \mathcal{N}\left(0, \sigma_{s, i}^{2}\right)$. Similarly the process/motion model is based on $\left\{\mathbf{p}_{i-1, k}\right\}$, and the associated process noise $n_{i-1}^{r}$ is assumed to follow a Gaussian distribution $n_{i-1}^{r} \sim \mathcal{N}\left(0, \sigma_{r, i-1}^{2}\right)$. Therefore the prediction step is:

$$
\left\{\begin{array}{l}
\mathbf{p}_{i \mid i-1, k}=\mathbf{p}_{i-1, k}, \\
\sigma_{i \mid i-1}^{2}=\sigma_{i-1 \mid i-1}^{2}+\sigma_{r, i-1}^{2}
\end{array},\right.
$$

and measurement update is given as:

$$
\left\{\begin{array}{l}
\mathbf{p}_{i \mid i, k}=\mathbf{p}_{i \mid i-1, k}+k_{i}\left(\mathbf{p}_{i, k}-\mathbf{p}_{i \mid i-1, k}\right), \\
\sigma_{i \mid i}^{2}=\sigma_{i \mid i-1}^{2}-k_{i} \sigma_{i \mid i-1}^{2}
\end{array}\right.
$$

where:

$$
k_{i}=\frac{\sigma_{i \mid i-1}^{2}}{\sigma_{i \mid i-1}^{2}+\sigma_{s, i}^{2}} .
$$

This results in the filtered set of points $\left\{\mathbf{p}_{i \mid i, k}\right\}$ which constitutes $\mathcal{V}_{i}^{f}$. 


\section{Experiments and Results}

The quality of VI-KinectDeform is analyzed both quantitatively and qualitatively. We use the "Facecap" dataset which captures a person's face deforming non-rigidly due to changing expressions in different scenes [23]. The selected scene includes 40 frames. We simulate a depth camera in V-Rep [1], placed approximately at $0.5 \mathrm{~m}$ away from the object and add Gaussian noise with zero mean and standard deviations of $0.01 \mathrm{~m}$, $0.03 \mathrm{~m}$ and $0.05 \mathrm{~m}$, respectively. Experiments are carried out using these datasets for both VI-KinectDeform and KinectDeform. A bilateral filter is used in the pre-processing step to obtain improved registration for both methods [22]. We use the algorithm proposed by Destelle et al. [7] for non-rigid registration in both methods. We use the proposed automated fusion scheme in both VI-KinectDeform and KinectDeform by replacing the empirical fusion scheme used previously. Post-processing is based on the bilateral mesh de-noising with very small parameters for the neighborhood size and the projection distance for both VI-KinectDeform and KinectDeform [8].

The quantitative evaluation of VI-KinectDeform as compared to KinectDeform is reported in Fig. 2. It shows the root mean square error (RMSE) of the data enhanced with VI-KinectDeform, and the data enhanced with KinectDeform with respect to the ground truth data for different noise levels. These results show superior performance of VI-KinectDeform in terms of overall accuracy of 3D reconstructions as compared to KinectDeform. It is noted that the accuracy of the proposed technique is restricted by the accuracy of the considered non-rigid registration algorithm. We have tested our proposed VI-KinectDeform by using non-rigid registration parameters obtained from noise free data. Post-processing step is skipped in this case. The resulting curve in Fig. 2(a) shows a significant decrease in error when using VI-KinectDeform as compared to its earlier version. This is observed through all frames. The qualitative analysis presented in Fig. 3, corresponding to the noise level and results in Fig. 2(a), shows superior quality of 3D reconstructions obtained via VI-KinectDeform in terms of feature preservation and smoothness when compared to the results obtained via KinectDeform.

For further analysis of performance of the proposed technique, we use the "Swing" dataset [24]. We, again, simulate a depth camera in V-Rep placed approximately at $1.5 \mathrm{~m}$ away from the object and add Gaussian noise with zero mean and standard deviation of $0.0075 \mathrm{~m}$. We use 20 frames for this experiment. We analyze the performance of the proposed VI-KinectDeform with 3 other view-independent surface representation schemes. These representation schemes are based on finding the surface approximation with respect to each centroid belonging to the leaf nodes of $\mathcal{U}_{i}$ and $\mathcal{U}_{i-1}$ lying close to $\mathcal{V}_{i}$ and $\mathcal{V}_{i-1}^{r}$.

The first scheme is based on finding the closest points in local neighborhoods of the centroids. The second scheme is based on finding the weighted mean of all points lying in local neighborhoods of each centroid using the weighting scheme similar to the one used in (5). The third scheme fits tangent planes to points in local neighborhoods and finds the projections of the centroid on them. It is similar to the proposed scheme wherein the degree of the polynomial is fixed to one.

Quantitative and qualitative results are shown in Fig. 4 and Fig. 5, respectively. As expected, Fig. 4 shows that the closest point-based method is least accurate followed by the weighted mean-based method, the plane projection-based method, and the proposed 


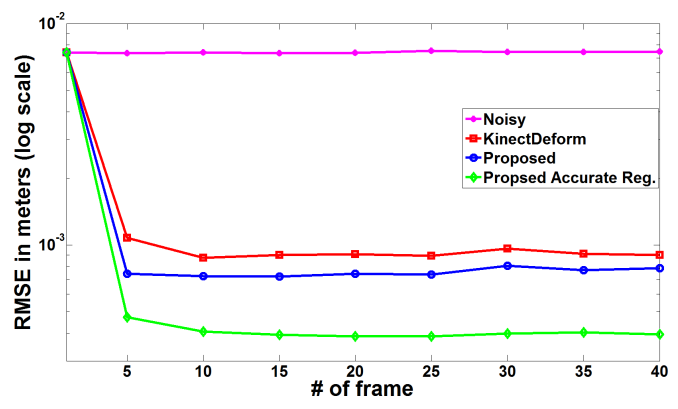

(a)

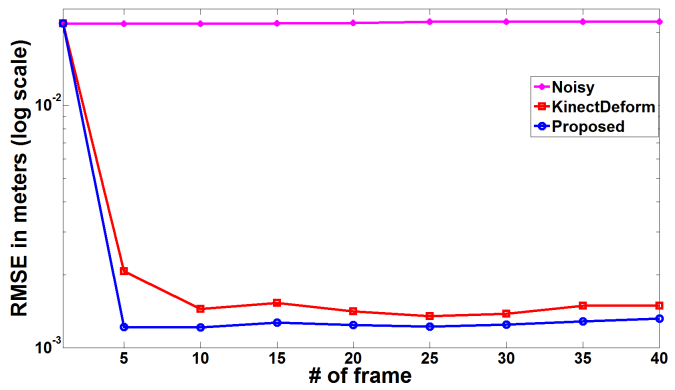

(b)

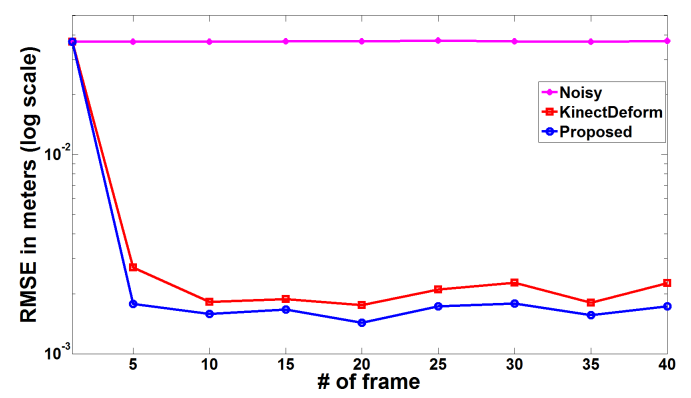

(c)

Fig. 2. "Facecap" dataset. Quantitative analysis on data with different levels of Gaussian noise. Each figure contains RMSE in log scale of: noisy data, result of KinectDeform and result of VIKinectDeform. (a) Results for Gaussian noise with standard deviation of $0.01 \mathrm{~m}$. It also contains RMSE in log scale of VI-KinectDeform with registration based on noise free data. (b) Results for Gaussian noise with standard deviation of $0.03 \mathrm{~m}$. (c) Results for Gaussian noise with standard deviation of $0.05 \mathrm{~m}$.

projection-based $M L S$ method in terms of overall accuracy. Similar results are obtained via quantitative analysis as shown in Fig. 5 wherein the proposed method produces the most accurate and feature preserving reconstruction. Plane projection-based method also gives good results but small features such as nose and curves on clothing are not well preserved. This experiment also shows that the proposed pipeline is generic enough 


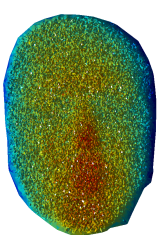

(a)



(f)

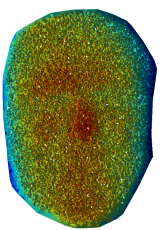

(k)

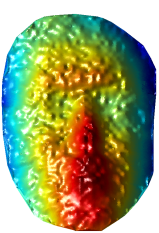

(b)

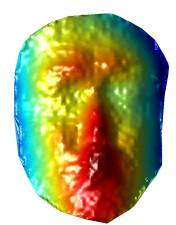

(g)

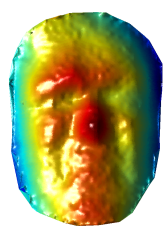

(1)

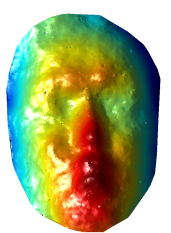

(c)

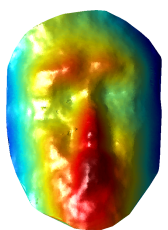

(h)

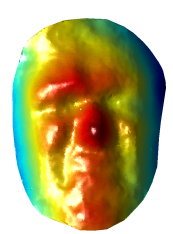

(m)

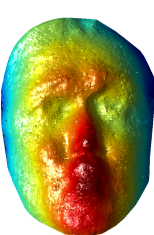

(d)

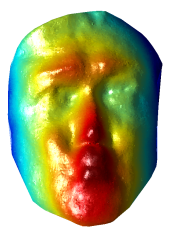

(i)

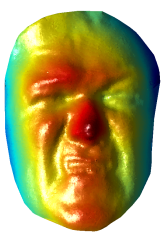

(n)

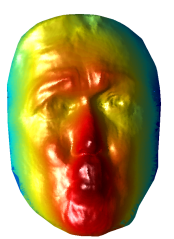

(e)

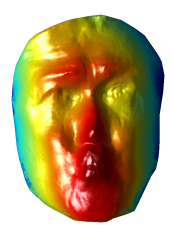

(j)

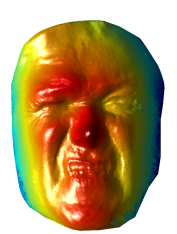

(o)

Fig. 3. "Facecap" dataset. First row: Frame \#5, Second row: Frame \#15, Third row: Frame \#35. Each row contains noisy data with Gaussian noise of standard deviation $0.01 \mathrm{~m}$, result of KinectDeform, result of VI-KinectDeform, result of VI-KinectDeform with registration based on noise free data and ground truth respectively.

such that any view-independent point-based surface representation scheme using local neighborhoods can replace the proposed $M L S$-based scheme.

\section{Conclusion and Future Work}

In this work we have proposed VI-KinectDeform, an automated recursive filtering scheme for producing enhanced 3D reconstructions of non-rigidly deforming objects. It improves upon our previous work, i.e., KinectDeform [2], by replacing the implicit viewdependent $T S D F$ based surface representation scheme with an explicit $M L S$-based viewindependent surface representation scheme [3]. This simplifies the pipeline by removing surface prediction and extraction steps. Moreover we improve upon the data fusion scheme by proposing an automated point tracking with a Kalman filter [10], The quantitative and qualitative evaluation of our method shows that it is able to produce smooth and feature preserving $3 \mathrm{D}$ reconstructions with an improved accuracy when compared to KinectDeform. We also show that the proposed pipeline is generic, and can use any view-independent point-based surface representation scheme. The generic and viewindependent nature of this algorithm allows for the extension to a multi-view system 


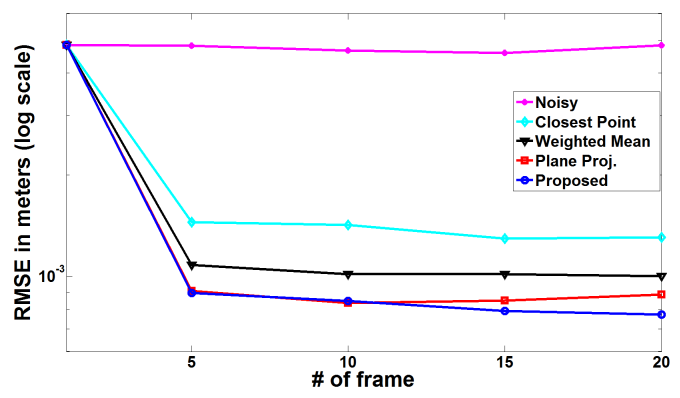

Fig. 4. "Swing" dataset. RMSE in log scale of: noisy data with Gaussian noise of standard deviation $0.0075 \mathrm{~m}$, result of closest point-based surface representation, result of weighted-mean based surface representation, result of local plane projection-based surface representation and result of the proposed projection-based $M L S$ surface representation. Please read Sect. 4 for more details.

to produce complete $360^{\circ}$ enhanced $3 \mathrm{D}$ reconstructions of scenes containing non-rigid objects. This constitutes our future work.

\section{Acknowledgment}

The "Facecap" dataset was provided courtesy of the Graphics, Vision \& Video Research Group of the Max Planck Institute for Informatics [23]. This work was supported by the National Research Fund (FNR), Luxembourg, under the CORE project C11/BM/1204105/FAVE/Ottersten.

\section{References}

1. V-REP, http://www.coppeliarobotics.com/

2. Afzal, H., Ismaeil, K.A., Aouada, D., Destelle, F., Mirbach, B., Ottersten, B.: KinectDeform: Enhanced 3D Reconstruction of Non-Rigidly Deforming Objects. In: 3DV Workshop on Dynamic Shape Measurement and Analysis. Tokyo, Japan (2014)

3. Alexa, M., Behr, J., Cohen-Or, D., Fleishman, S., Levin, D., Silva, C.T.: Computing and rendering point set surfaces. Visualization and Computer Graphics, IEEE Transactions on 9(1), 3-15 (Jan 2003)

4. Bylow, E., Sturm, J., Kerl, C., Kahl, F., Cremers, D.: Real-Time Camera Tracking and 3D Reconstruction Using Signed Distance Functions. In: Robotics: Science and Systems Conference (RSS) (June 2013)

5. Cheng, Z.Q., Wang, Y.Z., Li, B., Xu, K., Dang, G., Jin, S.Y.: A survey of methods for moving least squares surfaces. In: Proceedings of the Fifth Eurographics / IEEE VGTC Conference on Point-Based Graphics. pp. 9-23. SPBG'08, Eurographics Association, Aire-la-Ville, Switzerland, Switzerland (2008)

6. Cui, Y., Chang, W., Nll, T., Stricker, D.: KinectAvatar: Fully Automatic Body Capture Using a single Kinect. In: ACCV Workshop on Color Depth fusion in computer. ACCV (2012)

7. Destelle, F., Roudet, C., Neveu, M., Dipanda, A.: Towards a real-time tracking of dense point-sampled geometry. International Conference on Image Processing pp. 381-384 (2012) 


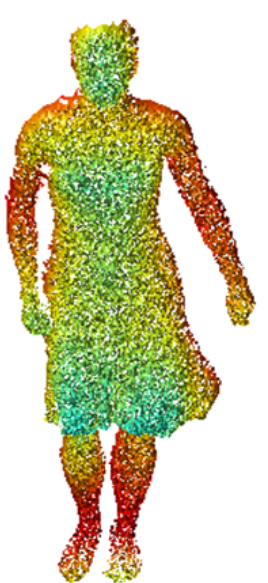

(a) Noisy

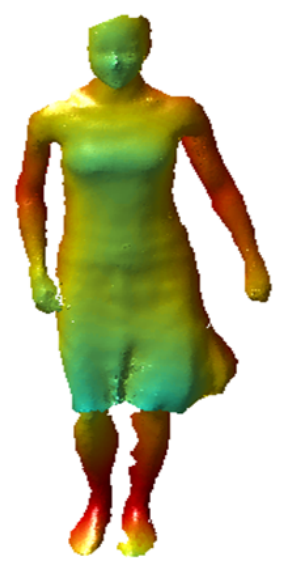

(d) Plane Proj.

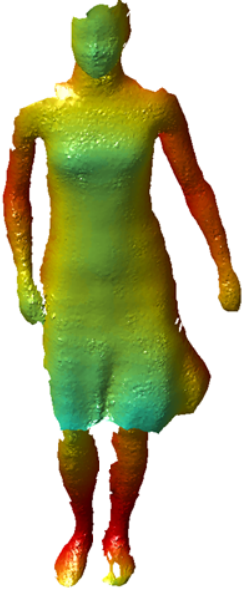

(b) Closest Point

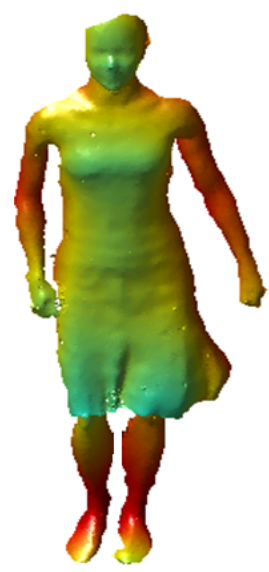

(e) Proposed Tech

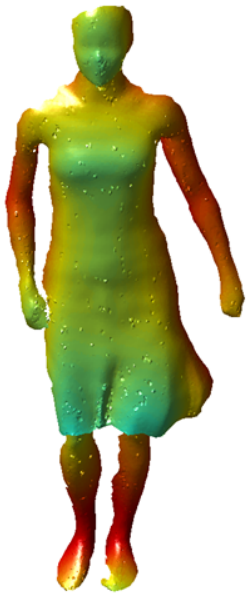

(c) Weighted Mean

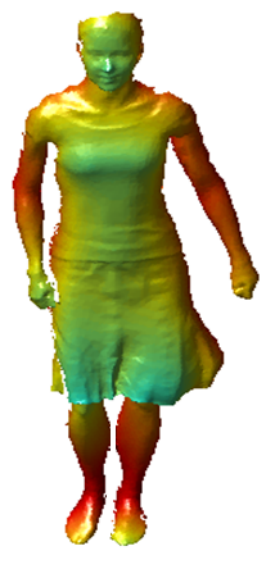

(f) Ground Truth

Fig. 5. "Swing" dataset. First row: Left: noisy data with Gaussian noise of standard deviation $0.0075 \mathrm{~m}$, Center: result of closest point-based surface representation, Right: result of weighted mean-based surface representation. Second row: Left: result of local plane projection-based surface representation, Center: result of the proposed projection-based $M L S$ surface representation, Right: ground truth.

8. Fleishman, S., Drori, I., Cohen-Or, D.: Bilateral mesh denoising. In: ACM SIGGRAPH 2003 Papers. pp. 950-953. SIGGRAPH '03, ACM, New York, NY, USA (2003)

9. Ismaeil, K.A., Aouada, D., Solignac, T., Mirbach, B., Ottersten, B.: Real-Time Non-Rigid Multi-Frame Depth Video Super-Resolution. In: CVPR Workshop on Multi-Sensor Fusion for Dynamic Scene Understanding. Boston, MA, USA (2015)

10. Kalman, R.E.: A new approach to linear filtering and prediction problems. Transactions of the ASME-Journal of Basic Engineering 82(Series D), 35-45 (1960) 
11. Levin, D.: Mesh-independent surface interpolation. In: Brunnett, H., Mueller (eds.) Geometric Modeling for Scientific Visualization. pp. 37-49. Springer-Verlag (2003)

12. Li, H., Adams, B., Guibas, L.J., Pauly, M.: Robust Single-view Geometry and Motion Reconstruction. In: ACM SIGGRAPH Asia 2009 Papers. pp. 175:1-175:10. SIGGRAPH Asia '09, ACM, New York, NY, USA (2009), http://doi.acm.org/10.1145/1661412.1618521

13. Mac Aodha, O., Campbell, N.D., Nair, A., Brostow, G.J.: Patch Based Synthesis for Single Depth Image Super-Resolution. In: ECCV (3). pp. 71-84 (2012)

14. Mrcio, C., Apolinario Jr., A.L., Souza, A.C.S.: KinectFusion for Faces: Real-Time 3D Face Tracking and Modeling Using a Kinect Camera for a Markerless AR System. SBC Journal on 3D Interactive Systems 4, 2-7 (2013)

15. Newcombe, R.A., Izadi, S., Hilliges, O., Molyneaux, D., Kim, D., Davison, A.J., Kohli, P., Shotton, J., Hodges, S., Fitzgibbon, A.: KinectFusion: Real-Time Dense Surface Mapping and Tracking. In: Proceedings of the 2011 10th IEEE International Symposium on Mixed and Augmented Reality. pp. 127-136. ISMAR '11, IEEE Computer Society, Washington, DC, USA (2011), http://dx.doi.org/10.1109/ISMAR.2011.6092378

16. Nießner, M., Zollhöfer, M., Izadi, S., Stamminger, M.: Real-time 3D Reconstruction at Scale using Voxel Hashing. ACM Transactions on Graphics (TOG) (2013)

17. Roth, H., Vona, M.: Moving Volume KinectFusion. In: Proceedings of the British Machine Vision Conference. pp. 112.1-112.11. BMVA Press (2012)

18. Rouhani, M., Sappa, A.: Non-rigid shape registration: A single linear least squares framework. In: Fitzgibbon, A., Lazebnik, S., Perona, P., Sato, Y., Schmid, C. (eds.) Computer Vision ECCV 2012, Lecture Notes in Computer Science, vol. 7578, pp. 264-277. Springer Berlin Heidelberg (2012)

19. Schuon, S., Theobalt, C., Davis, J., Thrun, S.: LidarBoost: Depth Superresolution for ToF 3D Shape Scanning. In Proc. of IEEE CVPR 2009 (2009)

20. Steinbrcker, F., Kerl, C., Cremers, D.: Large-Scale Multi-resolution Surface Reconstruction from RGB-D Sequences. In: Proceedings of the 2013 IEEE International Conference on Computer Vision. pp. 3264-3271. ICCV'13, IEEE Computer Society, Washington, DC, USA (2013), http://dx.doi.org/10.1109/ICCV.2013.405

21. Sturm, J., Bylow, E., Kahl, F., Cremers, D.: CopyMe3D: Scanning and printing persons in 3D. In: German Conference on Pattern Recognition (GCPR). Saarbrücken, Germany (September 2013)

22. Tomasi, C., Manduchi, R.: Bilateral Filtering for Gray and Color Images. In: Proceedings of the Sixth International Conference on Computer Vision. pp. 839-. ICCV '98, IEEE Computer Society, Washington, DC, USA (1998)

23. Valgaerts, L., Wu, C., Bruhn, A., Seidel, H.P., Theobalt, C.: Lightweight Binocular Facial Performance Capture Under Uncontrolled Lighting. ACM Trans. Graph.

24. Vlasic, D., Baran, I., Matusik, W., Popović, J.: Articulated mesh animation from multi-view silhouettes. In: ACM SIGGRAPH 2008 Papers. pp. 97:1-97:9. SIGGRAPH '08, ACM, New York, NY, USA (2008)

25. Wang, K., Wang, X., Pan, Z., Liu, K.: A two-stage framework for 3d facereconstruction from rgbd images. Pattern Analysis and Machine Intelligence, IEEE Transactions on 36(8), 1493-1504 (Aug 2014)

26. Zeng, M., Zheng, J., Cheng, X., Jiang, B., Liu, X.: Dynamic human surface reconstruction using a single kinect. In: Computer-Aided Design and Computer Graphics (CAD/Graphics), 2013 International Conference on. pp. 188-195 (Nov 2013)

27. Zollhöfer, M., Nießner, M., Izadi, S., Rehmann, C., Zach, C., Fisher, M., Wu, C., Fitzgibbon, A., Loop, C., Theobalt, C., Stamminger, M.: Real-time Non-rigid Reconstruction using an RGB-D Camera. ACM Transactions on Graphics (TOG) (2014) 